Published in the Nuclear Instruments and Methods, 127, 475 - 485, (1975)

BEAM-CAVITY INTERACTION IN ELECTRON

STORAGE RINGS

Eberhard Keil, Claudio Pellegrini, Angelo Turrin, and Andrew M. Sessler

April 1, 1975

Prepared for the U. S. Energy Research and

Development Administration under Contract W-7405-ENG-48

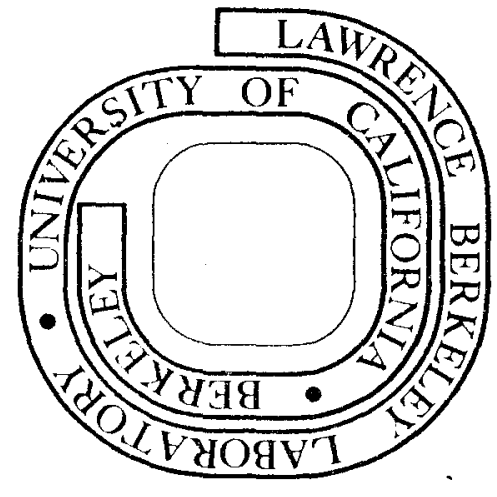

TWO-WEEK LOAN COPY

This is a Library Circulating Copy which may be borrowed for two weeks. For a personal retention copy, call Tech. Info. Division, Ext. 5545 
Reproduced with permission of North-Holland Publishing Company, Amsterdam, The Netherlands, November 10, 1975.

NUCLEAR INSTRUMENTS AND METHODS $127(1975)$ 475-485; C NORTH-HOLLAND PUBLISHING CO.

\title{
BEAM-CAVITY INTERACTION IN ELECTRON STORAGE RINGS
}

\author{
EBERHARD KEIL* \\ European Organization for Nuclear Research, Genera, Suitzerland \\ CLAUDIO PELLEGRINI ${ }^{\dagger}+$ and ANGELO TURRIN ${ }^{\dagger}$ \\ Laboratori Nazionali di Frascati, Frascati (Roma), Italy \\ and \\ ANDREW M. SESSLER** \\ Lawrence Berkeley Laboratory, University of California, Berkeley, California 94720 , U.S.A.
}

Received I April 1975

A formal expression is obtained for the energy loss per turn, of a rigid bunch of electrons, to a closed cylindrical cavity with quality factor $Q$. The expression is valid provided the diameter of the entrance and exit ports for the beam are small compared to the bunch length. The effect of the ports is studied in an independent computational method. The energy loss is numerically evaluated for a range of parameters of interest to electron storage rings.

\section{Introduction}

The next generation of electron storage rings, which currently is under design, Super-Adone ${ }^{1}$ ), PEP $^{2}$ ), EPIC $^{3}$ ), PETRA $^{4}$ ), and the electron option of ISABELLE ${ }^{5}$ ), has in typical cases electron bunches of about $0.1 \mathrm{~m}$ length and peak currents of up to $1000 \mathrm{~A}$. For these devices, beam-loading of the rf system must be carefully considered ${ }^{6}$ ).

Of course, the loading of an rf cavity by a beam is an old and well-understood subject. However, in the classical cases the bunch spacing is equal to an $\mathrm{rf}$ wavelength and the bunch charge is so small that the energy loss is small compared to the energy gained by the bunch from the excited cavity. A striking exception to this situation is envisaged in the rf accelerating structure of an electron ring accelerator, where a bunch of negligible extent, and with very high charge, is expected to lose a considerable amount of energy. In fact, only the finite radius of the beam tube and the periodicity of the structure keep the loss within bounds ${ }^{7}$ ).

The situation in electron storage rings is between these two extremes: The bunch charge is high, but the bunch length is of the order of the rf cavity length. In contrast to the electron ring accelerator, the bunch passes repeatedly through the same cavity.

We employ routine procedures to evaluate the energy loss of an electron bunch to a model rf cavity, namely to a closed cylindrical lossy cavity. This model should be valid provided the bunch is long compared to the diameter of the entrance and exit ports of the cavity. In addition, we study, by a numerical method, the effect of the ports on the energy loss.

In section 2, we present a formal expression for the energy loss in a structure characterized by an arbitrary coupling impedance. In section 3 we introduce the expression of $Z(\omega)$ for a pill-box cavity and obtain a formula for the energy loss as a double sum over all the cavity resonant modes. Numerical results are obtained and discussed in section 4 .

\section{Definition of the energy loss for an arbitrary coupling impedance}

Let $\varepsilon_{l}(n)$ and $\phi_{t}(n)$ be the energy displacement and the azimuthal position of the th particle at the $n$th revolution, with respect to the synchronous particle, having energy $E$. The equations of motion can be written as:

$$
\phi_{l}(n)-\phi_{l}(n-1)=\frac{2 \pi \alpha}{E} \varepsilon_{l}(n),
$$

\footnotetext{
* Present address: SLAC, P. O. Box 4349, Stanford, California 94305, U.S.A.

+ Work supported by CNEN.

+ On lcave to JET Group, Culham Laboratory, Abingdon, Berkshire OX143DB, United Kingdom.

** Work supported by the U. S. Energy Research and Development Administration.
} 


$$
\varepsilon_{l}(n+1)-\varepsilon_{l}(n)=-\beta \varepsilon_{l}(n)-q_{l}(n)+e V_{0}\left[T \phi_{l}(n) / 2 \pi\right]+\text { energy change due to self-fields, }
$$

where $\alpha$ is the momentum compaction factor, $T$ is the revolution period, $V_{0}(t)$ is the external radio-frequency voltage, $\beta$ is the damping coefficient produced by synchrotron radiation and $q_{1}(n)$ is the energy loss at the $n$th revolution due to incoherent synchrotron radiation. Let $I(t)$ be the longitudinal beam current, $I(\omega)$ its Fourier transform and $Z(\omega)$ the longitudinal coupling impedance. The azimuthal component of the self-electric field can be written $\operatorname{as}^{8}$ ):

$$
2 \pi R \varepsilon(t)=-\int \mathrm{d} \omega Z(\omega) I(\omega) \exp (-\mathrm{i} \omega t)
$$

The energy loss of the $l$ th particle at the $n$th revolution is then given by:

$$
\xi_{l}(n)=-e \int \mathrm{d} \omega Z(\omega) I(\omega) \exp \left\{-i \omega\left[n T+T \phi_{l}(n) / 2 \pi\right]\right\} .
$$

We write the beam current as:

$$
I(l)=e \sum_{m=-\infty}^{+\infty} \sum_{k=1}^{N} \delta\left[t-m T-T \phi_{l}(n) / 2 \pi\right],
$$

where $N$ is the number of particles, and the sum over $m$ describes different revolutions.

Defining $l(\omega)$ as:

$$
I(\omega)=\frac{1}{2 \pi} \int I(t) \exp (\mathrm{i} \omega t) \mathrm{d} t,
$$

using eq. (4) and substituting in eq. (3), we obtain:

$$
\begin{aligned}
\check{\zeta}_{l}(n)= & -\frac{e^{2}}{2 \pi} \sum_{m} \int \mathrm{d} \omega Z(\omega) \exp \left\{-\mathrm{i} \omega(n-m) T-\frac{\mathrm{i} \omega T}{2 \pi}\left[\phi_{l}(n)-\phi_{l}(m)\right]\right\}- \\
& -\frac{e^{2}}{2 \pi} \sum_{m} \sum_{k \neq l} \int \mathrm{d} \omega Z(\omega) \exp \left\{-\mathrm{i} \omega(n-m) T-\frac{\mathrm{i} \omega T}{2 \pi}\left[\phi_{l}(n)-\phi_{k}(m)\right]\right\} .
\end{aligned}
$$

The first term on the right-hand side of eq. (5) gives the incoherent energy loss and in the following will be neglected; the second term describes the coherent energy loss. The causality condition requires that in the expression:

$$
\xi_{l}(n)=-\frac{e^{2}}{2 \pi} \sum_{m=-\infty}^{+\infty} \sum_{k \neq 1} \int \mathrm{d} \omega Z(\omega) \exp \left\{-i \omega T\left[n-m+\frac{\phi_{l}(n)-\phi_{k}(m)}{2 \pi}\right]\right\},
$$

the only non-zero contributions must come from those terms satisfying the relationship:

$$
n-m+\frac{\phi_{l}(n)-\phi_{k}(m)}{2 \pi}>0 .
$$

Hence the function $Z(\omega)$ must be analytic except for poles in the lower half complex $\omega$-plane.

In the following we will assume the bunch to be much shorter than the machine circumference, or $\phi_{k} / 2 \pi \ll 1$. We can then rewrite the expression (6) for the coherent energy loss as:

$$
\begin{aligned}
\xi_{l}(n)= & -\frac{e^{2}}{2 \pi} \sum_{m<n} \sum_{k \neq 1} \int \mathrm{d} \omega Z(\omega) \exp \left\{-i \omega T\left[n-m+\frac{\phi_{l}(n)-\phi_{k}(m)}{2 \pi}\right]\right\}- \\
& -\frac{e^{2}}{2 \pi} \sum_{k \neq 1} \int \mathrm{d} \omega Z(\omega) \exp \left\{-\mathrm{i} \omega T \frac{\phi_{l}(n)-\phi_{k}(n)}{2 \pi}\right\} S\left[\phi_{l}(n)-\phi_{k}(n)\right],
\end{aligned}
$$

where $S(x)$ is the step function. 
The first term on the right hand side of eq. (8) describes the "retarded energy loss" due to the fields induced in the cavity on the revolutions previous to the $n$th revolution, while the second term describes the "instantaneous energy loss of the /th particle".

The coherent energy loss, as defined in eq. (8), depends on the position of the /th particle inside the bunch. A quantity of more practical interest is the average energy loss per particle:

$$
W(n)=\frac{1}{N} \sum_{l} \xi_{l}(n)
$$

If the particle distribution function is assumed to be time-independent, the average energy loss, $W$, will not depend on $n$. In the following, we shall only consider this case.

\section{The closed-cylindrical-cavity impedance}

The coupling impedance $Z(\omega)$ must be evaluated for any given structure for which we wish to compute the beam-structure interaction. For a closed cylindrical cavity, this is done in appendices A and B. Eqs. (60) and (63) are analytical expressions for the energy loss $W$. For our present purpose, it is convenient to rewrite them in terms of an effective impedance $Z_{\text {crf }}$,

$$
W=-e I_{\mathrm{iv}} Z_{\mathrm{c} \cdot \mathrm{f}}
$$

and an average beam current $I_{\mathrm{av}}$,

$$
I_{\mathrm{av}}=e N / T=e \omega_{0} N / 2 \pi
$$

Here, $\omega_{0}=2 \pi / T$ is the circular bunch frequency, and $N$ is the bunch population. From eqs. (10), (11) and (60) or (63), two equivalent expressions for $Z_{\text {eff }}$ may be obtained, both of them valid for a Gaussian bunch shape, as given by eq. (58), with an rms half-length $\Delta$. The first expression, which exhibits the periodic nature of the beam current driving the cavity, is:

$$
Z_{\mathrm{eff}}=\sum_{n=0}^{\infty} Z_{n} \exp \left[-\frac{n^{2} \omega_{0}^{2} \Delta^{2}}{v^{2}}\right]
$$

Here, $Z_{n}$ is the cavity impedance at the $n$th harmonic of the revolution frequency, given by:

$$
Z_{n}=\frac{16 \omega_{0}^{2}}{b g v^{2} c} \sum_{p=0}^{\infty} \frac{n^{2}}{1+\delta_{p 0}} \frac{1-(-1)^{n} \cos \left(n \omega_{0} g / v\right)}{\left[(\pi p / g)^{2}-\left(n \omega_{0} / v\right)^{2}\right]^{2}}\left(\frac{n \omega_{0}}{8 \pi \sigma}\right)^{\frac{1}{2}} \frac{1}{D_{n p}} .
$$

Here, $b$ and $g$ are the cavity radius and length, $v$ and $c$ are the beam and light velocities, $\sigma$ is the cavity-wall conductivity and $D_{n p}$ is defined in eq. (61). The form (12) of the effective impedance will be used for studying the effect of the beam ports.

The second form, obtained from eq. (63), exhibits the resonant properties of the cavity:

$$
Z_{\mathrm{eff}}=\frac{16 \pi}{g \omega_{0}} \sum_{p=0}^{\infty} \sum_{s=1}^{\infty} \frac{R_{s p} \exp \left(-\omega_{s p}^{2} \Delta^{2} / v^{2}\right)}{\left(1+\delta_{p 0}\right) v_{s}^{2} J_{i}^{2}\left(v_{s}\right)} \frac{1-(-1)^{p} \cos \left(\omega_{s p} g / v\right)}{\left\{1+\left[(p \pi b) /\left(\gamma g v_{s}\right)\right]^{2}\right\}^{2}}
$$

In eq. (14), $v_{s}$ is defined as the $s$ th root of $J_{0}\left(v_{s}\right)=0, \gamma$ is the electron energy in units of its rest energy, and the resonant frequency $\omega_{s p}$ of the $E_{0 s p}$ mode is given by:

$$
\left(\omega_{s p} / c\right)^{2}=\left(v_{s} / b\right)^{2}+(\pi p / g)^{2}
$$

The "resonant term" $R_{s p}$ is:

$$
R_{s p}=\frac{\sinh \left(\pi \omega_{s p} / \omega_{0} Q_{s p}\right)}{\cosh \left(\pi \omega_{s p} / \omega_{0} Q_{s p}\right)-\cos \left(2 \pi \omega_{s p} / \omega_{0}\right)}
$$

and the quality factor $Q_{s p}$ is:

$$
Q_{s p}=(b / c)\left(2 \pi \sigma \omega_{s p}\right)^{\frac{1}{2}} .
$$


The resonant term $R_{s p}$ describes the effect of the multiple passages of the bunch through the cavity. It was also derived by Wilson ${ }^{9}$ ) using a different technique. Because of the oscillatory behaviour of the denominator, it is a rapidly varying function of $\omega_{0}$, in particular when $\omega_{s p} \gg \omega_{0}$. Upper and lower bounds for $R_{s p}$ can be found by inserting:

$$
\cos \left(2 \pi \omega_{s p} / \omega_{0}\right)= \pm 1
$$

into eq. (16):

$$
\check{R}_{s p}=\tanh \left(\pi \omega_{s p} / 2 \omega_{0} Q_{s p}\right) \leq R_{s p} \leq \operatorname{coth}\left(\pi \omega_{s p} / 2 \omega_{0} Q_{s p}\right)=\hat{R}_{s p} .
$$

Averaging $R_{s p}$ over one period of $\cos \left(2 \pi \omega_{s p} / \omega_{0}\right)$ yields $\left.{ }^{10}\right)$ :

$$
\left\langle R_{s p}\right\rangle=1 \text {. }
$$

These relations will be used later on in the discussion of numerical results.

In the limit $Q_{s p} \rightarrow \infty$, we have $R_{s p} \rightarrow 0$, unless the resonant condition $\omega_{s p} / \omega_{0}=h$ is satisfied, where $h$ is an integer. In this case, one has, for $Q_{s p} \gg h$ :

$$
R_{s p} \approx 2 Q_{s p} /(\pi h)
$$

Hence, for a perfectly conducting cavity, there is no energy loss, unless the resonance condition $\omega_{s p} / \omega_{0}=\mathbf{h}$ is satisfied.

In evaluating $Z_{\text {eff }}$ from eq. (12) or (14) one must distinguish two cases. For externally driven rf cavities, the term with $p=0$ and $s=1$ should be removed from the sum. It corresponds to the driven fundamental mode of the cavity and needs a separate treatment ${ }^{11}$ ). For cavities which are not driven from an external rf power source and which might exist in an electron storage ring, the full formulae (12) and (14) may be used.

\section{Numerical evaluation of $Z_{\text {eff }}$}

In this section estimates of $Z_{\mathrm{eff}}$ will be obtained for values of the parameters relevant to electron storage rings. To this end, it is convenient to rewrite $Z_{\text {eff }}$ in terms of 4 scaled variables: $h, \lambda, \mu$, and $Q_{1 u}$, defined as follows:

$$
\begin{aligned}
h & =\omega_{10} / \omega_{0}, \\
\lambda & =g / b, \\
\mu & =\Delta / b, \\
Q_{10} & =(b / c)\left(2 \pi \sigma \omega_{10}\right) .
\end{aligned}
$$

We shall call $h$ the harmonic number, $\lambda$ the cavity aspect ratio, $\mu$ the normalized bunch length, and $Q_{10}$ the quality factor of the $E_{010}$ mode.

In these new parameters, the effective impedance of eq. (14) becomes, when converted to mks units (by multiplying by $Z_{0} c / 4 \pi$, where $Z_{0}=120 \pi \Omega$ is the impedance of free space):

$$
Z_{\mathrm{eff}}=\frac{4 Z_{0} h}{\lambda v_{1}} \sum_{p=0}^{\infty} \frac{\exp \left[-(\pi p \mu / \lambda)^{2}\right]}{1+\delta_{p 0}} \sum_{s=1}^{\infty} R_{s p} \frac{1-(-1)^{p} \cos \left\{(\lambda / \beta)\left[v_{s}^{2}+(\pi p / \lambda)^{2}\right]^{\frac{1}{2}}\right\}}{v_{s}^{2} J_{1}^{2}\left(v_{s}\right)} \frac{\exp \left[-\left(v_{s} \mu\right)^{2}\right]}{\left[1+\left(\pi p / \gamma v_{s} \lambda\right)^{2}\right]^{2}} .
$$

Here, $\beta=v / c$. In the new variables, we also have:

$$
\begin{aligned}
& R_{s p}=\frac{\sinh \delta_{s p}}{\cosh \delta_{s p}-\cos \left(2 Q_{s p} \delta_{s p}\right)} \\
& \delta_{s p}=\frac{\pi h}{v_{1} Q_{s p}}\left[v_{s}^{2}+(\pi p / \lambda)^{2}\right]^{\frac{\xi}{2}} \\
& Q_{s p}=Q_{10} v_{1}^{-\frac{1}{2}}\left[v_{s}^{2}+(\pi p / \lambda)^{2}\right]^{\frac{1}{4}}
\end{aligned}
$$


The effective impedance (25) was evaluated on a computer. As one might have suspected from the arguments given above, the resonant factors $R_{s p}$ are varying too rapidly to yield smooth curves for $Z_{\mathrm{eff}}$. We have therefore decided to present only curves for the minimum, average, and maximum impedance, using the values of $R_{s p}$ given in eqs. (18) and (19). It may be seen from eq. (26) that in this case $Z_{\text {eff }}$ only depends on the ratio $h / Q_{10}$. Fig. I shows the results obtained for a range of values for $\lambda$ and $\mu$. The average impedance divided by the harmonic number $\left\langle Z_{\text {eff }}\right\rangle / h$, which is independent of $Q_{10}$ and $h$, is shown for a wider range of $\lambda$ and $\mu$ and for two cases: fig. 2 shows $\left\langle Z_{\text {crf }}\right\rangle / h$ for an active cavity when the $E_{010}$ mode is not included in the summation, and fig. 3 shows $\left\langle Z_{\text {ar }}\right\rangle / h$ for a passive cavity where the $E_{010}$ mode is included. A comparison between figs. 2 and 3 shows that for long cavities $(\lambda \geqslant 1)$ the effective impedances are practically the same for both cases, while for short cavities $(\lambda \ll 1)$ leaving out the $E_{010}$ mode reduces $\left\langle E_{\text {cif }}\right\rangle$ by a large factor, as one would expect.

The effect of the beam entrance and exit ports on the coupling impedance has been studied by a computational procedure ${ }^{12}$ ) for finding the coupling impedance of the infinite structure shown in fig. 4 at all the harmonics of (1) 1 , and summing over them according to eq. (12). Two new parameters appear in this calculation: $a / b$, the ratio helween hole and cavity radius, and $d / h$, the ratio between the cavity spacing and the cavity radius. We made $d$ equal to one rf wavelength. We have verified that changing $d / b$ has little influence on the effective impedance.

Figs 5 and 6 show the results of this calculation in terms of "beam-port reduction factors". In order to obtain the impedance of a cavity with beam ports, the values shown in figs. 2 and 3 must be multiplied by the factors shown in figs. 6 and 7 , respectively. These factors are always smaller than unity. Hence, calculations neglecting the beam ports always overestimate the impedance. For $\alpha \ll \mu$, i.e., when the beam-port radius is small compared to the bunch length, the reduction factors are close to unity, and the error committed in neglecting the beam ports is small. Substantial reductions are obtained when the beam-port radius becomes bigger than the bunch length.

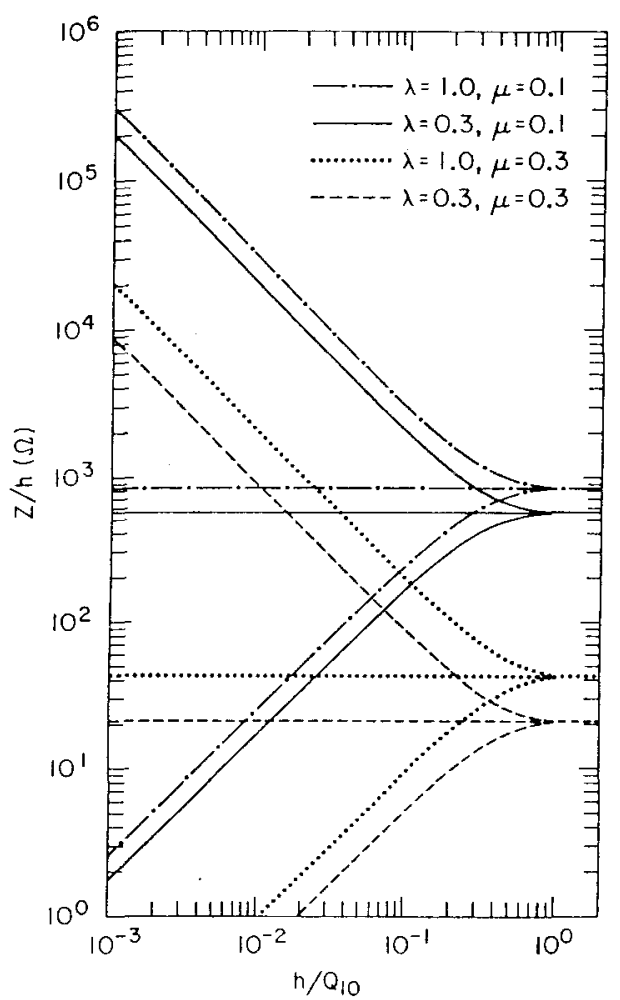

Fig. 1. Minimum, average, and maximum impedance of an active closed cylindrical cavity. $\lambda$ is cavity length/cavity radius, $\mu$ is bunch length/cavity radius, $h$ is the harmonic number, and $Q_{10}$ is the quality factor of the $E_{010}$ mode. The impedance of the $E_{010}$ mode is not included.

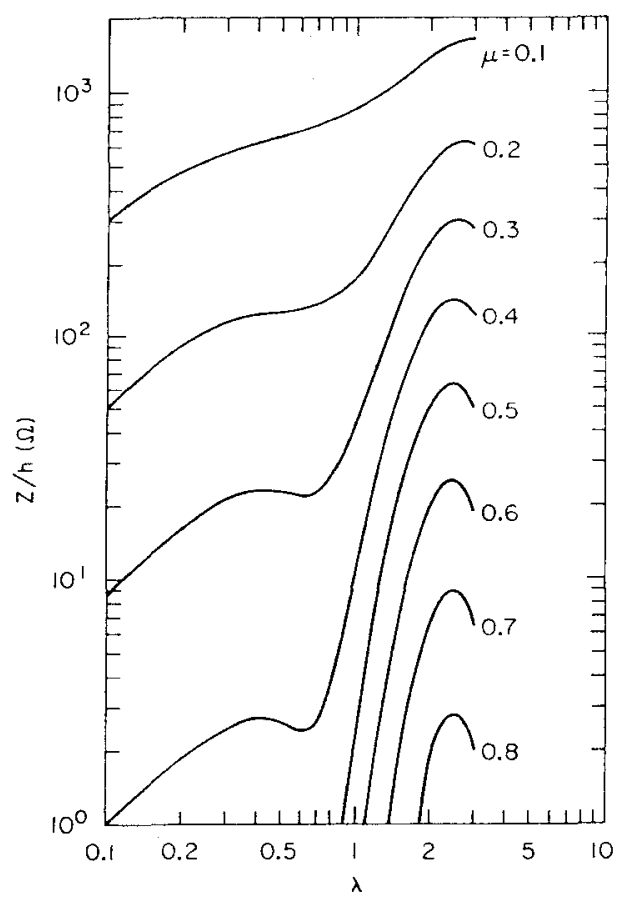

Fig. 2. Average impedance of an active closed cylindrical cavity. The contribution of the E010 mode is not included. 
Fig. 7 shows a specific example: the average impedance of the PEP rf system ${ }^{2}$ ). The cavity dimensions are $a=6.4 \mathrm{~cm}, b=32 \mathrm{~cm}, g=22.4 \mathrm{~cm}$; their number is 90 . The design current is $82 \mathrm{~mA}$ in three bunches, and hence $h=864$. At the design bunch length, $\Delta=2.2 \mathrm{~cm}$, the total energy loss due to higher modes is about $10 \mathrm{MeV}$ if the beam ports are neglected, and about $2.5 \mathrm{MeV}$ if they are taken into account.

\section{Conclusions}

We have evaluated the energy loss of a bunched beam passing through a closed cylindrical lossy cavity in an electron storage ring. Our results are given in a series of graphs. They show that in typical cases the energy

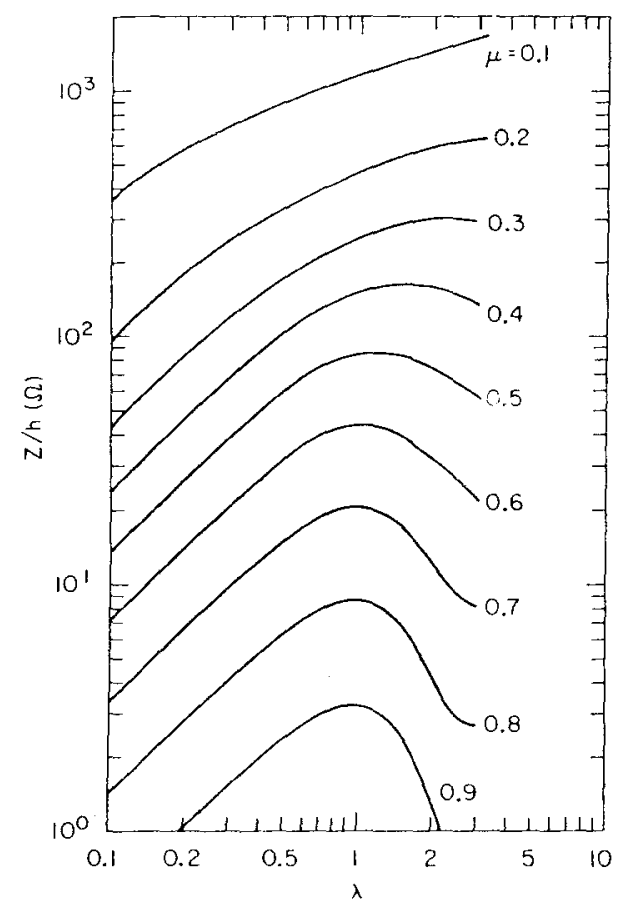

Fig. 3. Average impedance of a passive closed cylindrical cavity. The contribution of the $E_{010}$ mode is included.

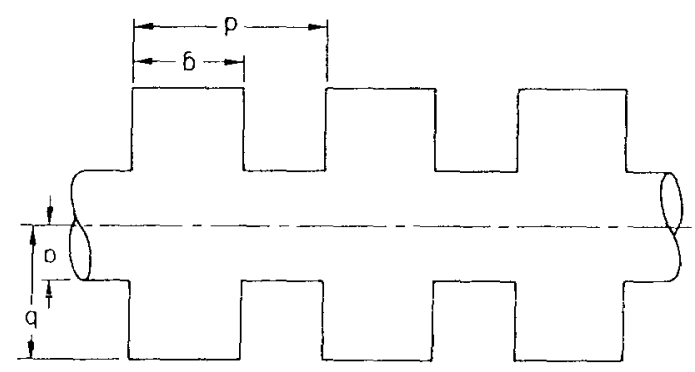

Fig. 4. Geometry of the infinite structure used for calculating the effect of the beam ports.

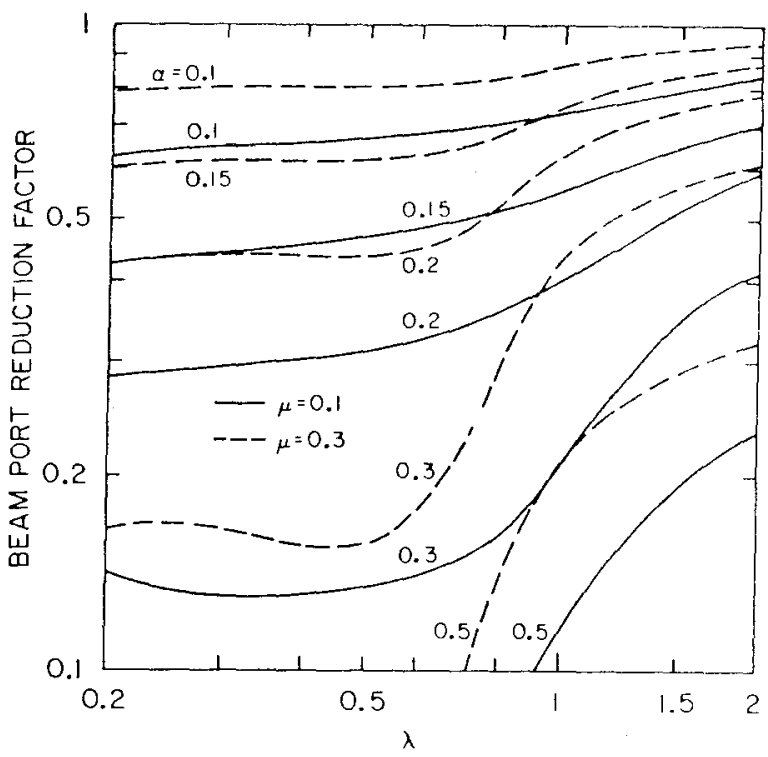

Fig. 5. Beam-port reduction factor for an active cavity, $x=a / b$ is the ratio of the beam-port and cavity radii. The contribution of the $E_{010}$ mode is not included

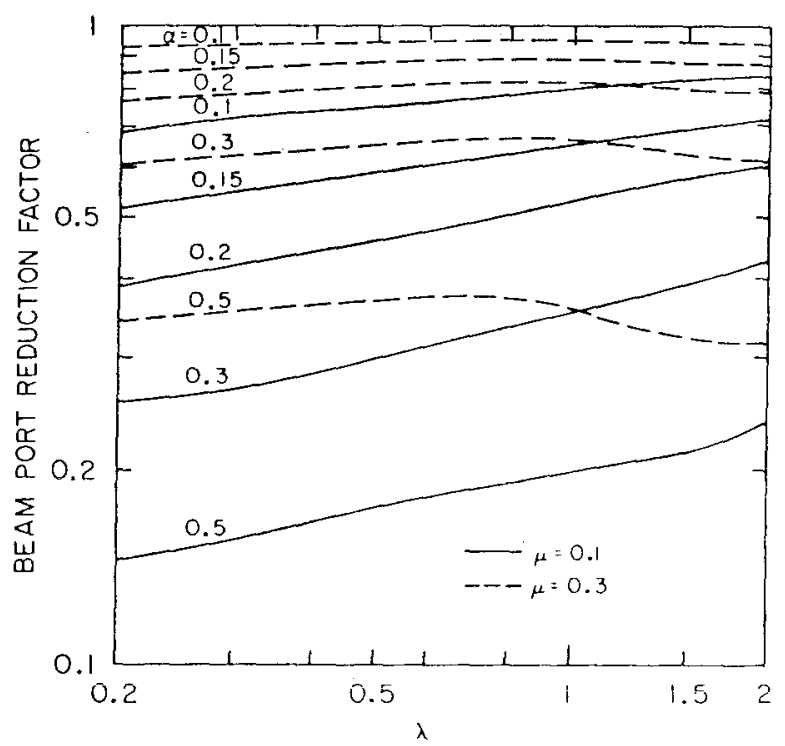

Fig. 6. Beam-port reduction factor for a passive cavity. The contribution of the $E_{010}$ mode is included. 


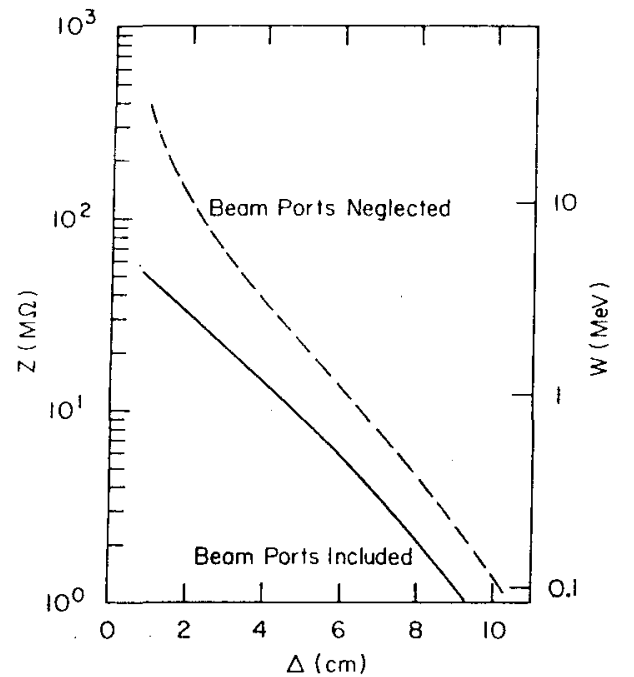

Fig. 7. Average impedance and energy loss due to the PEP rf system. The contribution of the $E_{010}$ mode is not included.

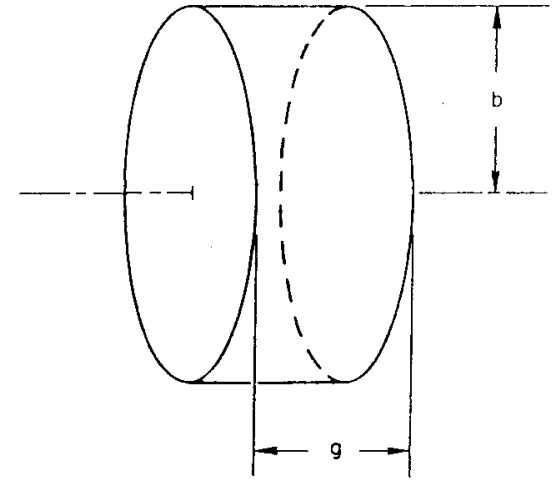

Fig. 8. Geometry of a closed cylindrical cavity.

loss due to the excitation of the cavities adds a significant amount to the energy loss due to synchrotron radiation. Therefore the rf system of these storage rings must be designed to handle this additional power loss, by increasing the rf voltage and hence the total if power installed beyond what would have been necessary to handle synchrotron radiation alone.

Our calculation has been done for machines with either a single bunch in the beam, or with several equidistant bunches with equal populations. It could easily be extended to cases with unequal bunches with unequal spacings, or to the case of two counter-rotating beams.

Since the beam-cavity interaction produces such a large coherent energy loss, it must be expected that it strongly affects the synchrotron motion ${ }^{13}$ ), as well as other phenomena such as bunch lengthening.

The authors are grateful to H.G. Hereward for carefully reading the manuscript and pointing out a number of small errors in the first version, and to R. A. Early (SLAC) and A. Kenney (LBL) for their help with the computations.

\section{References}

1) Superadone Design Study, INFN (1974).

$\Rightarrow$ Report LBL-2688/SLAC-171 (1974).

3) G. H. Rees et al., Proc. IXth Intern. Conf. on High energy accelerators (Stanford, 1974) p. 548.

4) Petra Proposal, DESY (1974).

5) Report BNL 18891 (1974),

i) Y. Orlov (Yerevan), private communication, 1971; P. L. Morton (SLAC), private communication, 1973.

7) E. Keil, ISR/TH/69-49 (1969); and Proc. VIlth Intern. Conf. on High energy accelerators (Yerevan, 1969; Academy of Sciences of Armenia SSR, 1970) vol. II, p. 551.

8) A. M. Sessler, IEEE Trans. Nucl. Sci. NS-18, no. 3 (1971) 1039.

3) P. B. Wilson, Proc. IXth Intern. Conf. on High energy accelerators (Stanford, 1974) p. 57.

10) M. Sands, PEP-90. SLAC (unpublished).

11) P. L. Morton and V. K. Neil, Proc. Symp. Electron ring accelerators, LRL Report UCRL 18103 (1968) p. 365.

12) E. Keil and B. Zotter, Particle Accelerators 3 (1972) 11.

13) M. H. R. Donald, RHEL, Report RL-74-154 (1974),

\section{Appendix A}

Electromagnetic field in a closed resistive cylindrical cavity

The cavity considered is shown in fig. 8 . It has cylindrical symmetry around the $z$-axis, the beam direction, infinite conductivity on the walls perpendicular to $z$, and conductivity $\sigma$ on the wall parallel to $z$. Using cylindrical 
coordinates $r, \theta, z$, we assume that all derivatives with respect to $\theta$ vanish, and that the only non-zero component of the current density is:

$$
j_{z}=I(z-v t) \delta(r) / 2 \pi r
$$

where $I(z-v t)$ is the instantaneous current at position $z$ and time $t$.

Maxwell's equations are written in terms of the vector and scalar potentials $A, \phi$, using the Lorentz gauge. We obtain $A_{r}=A_{\theta}=0$, and:

$$
\begin{gathered}
\square A_{z}=-(4 \pi / c) j_{z}, \\
\square \phi=-4 \pi \rho, \\
\frac{\partial A_{z}}{\partial z}+\frac{1}{c} \frac{\partial \phi}{\partial t}=0 .
\end{gathered}
$$

The boundary conditions are:

$$
\begin{array}{ll}
E_{r}=0, & \text { for } z=0 \text { and } z=g, \\
\tilde{E}_{z}=\mathscr{h} \tilde{H}_{\theta}, & \text { for } \quad r=b .
\end{array}
$$

Here, $\widetilde{E}_{z}$ and $\widetilde{H}_{\theta}$ are the Fourier transforms of $E_{z}$ and $H_{\theta}$, respectively; i.e.,

$$
E_{z}(r, z, t)=\int \tilde{E}_{z}(r, z, \omega) \exp (-\mathrm{i} \omega t) \mathrm{d} \omega .
$$

The wall impedance:

$$
\mathscr{R}=(1-\mathrm{i})(\omega / 8 \pi \sigma)^{\frac{1}{2}},
$$

satisfies the condition:

$$
\mathscr{R}(-\omega)=\mathscr{R}^{*}(\omega)
$$

where the asterisk denotes the complex conjugate.

We now write $A_{z}$ and $\phi$ in the form:

$$
\begin{aligned}
& A_{z}=\sum_{p=0}^{\infty} \int A_{p}(\omega, r) \cos (\pi p z / g) \exp (-\mathrm{i} \omega t) \mathrm{d} \omega, \\
& \phi=\sum_{p=0}^{\infty} \int B_{p}(\omega, r) \sin (\pi p z / g) \exp (-\mathrm{i} \omega t) \mathrm{d} \omega,
\end{aligned}
$$

and obtain from eq. (32):

$$
B_{p}(\omega, r)=(i c / \omega)(\pi p / g) A_{p}(\omega, r) .
$$

Now, eq. (33) is automatically satisfied, and eqs. (30) and (34) are reduced to:

$$
\begin{gathered}
\frac{1}{r} \frac{\partial}{\partial r}\left(r \frac{\partial A_{p}}{\partial r}\right)+\lambda^{2} A_{p}=-\frac{8 \pi c_{p}(\omega)}{c g\left(1+\delta_{p 0}\right)} \frac{\delta(r)}{2 \pi r}, \\
A_{p}(\omega, b)=\left.\frac{i \omega}{c} \frac{\mathscr{R}}{\lambda^{2}} \frac{\partial A_{p}}{\partial r}\right|_{r=b},
\end{gathered}
$$

where:

$$
\dot{\lambda}^{2}=(\omega / c)^{2}-(\pi p / g)^{2},
$$


and:

$$
c_{p}(\omega)=\frac{1}{2 \pi} \int_{0}^{g} \mathrm{~d} z \int_{-\infty}^{+\infty} \exp (\mathrm{i} \omega t) \cos (\pi p z / g) I(z-v t) \mathrm{d} t .
$$

The solution of eq. (41) can be written as the sum of that solution of the homogeneous equation which is finite for $r=0$, and a solution of the inhomogeneous equation, chosen such as to describe outgoing waves at $r \rightarrow \infty$ :

$$
A_{p}=a_{p} J_{0}(\lambda r)+\frac{2 \pi \mathrm{i}}{c g\left(1+\delta_{p 0}\right)} H_{0}^{(1)}(\lambda r) c_{p}(\omega) .
$$

The factor $a_{p}$ is determined by eq. (42), which yields:

$$
A_{p}(\omega, r)=-\frac{2 \pi \mathrm{i} c_{p}(\omega)}{c g\left(1+\delta_{p 0}\right)}\left\{J_{0}(\lambda r) \frac{H_{0}^{(1)}(\lambda b)+(\mathrm{i} \omega / c \lambda) \mathscr{R} H_{1}^{(1)}(\lambda b)}{J_{0}(\lambda b)+(\mathrm{i} \omega / c \lambda) \mathscr{R} J_{1}(\lambda b)}-H_{0}^{(1)}(\lambda r)\right\} .
$$

\section{Appendix B}

Energy loss of particles crossing a cavity

Consider one of the charged particles in the beam, moving along the trajectory:

$$
\begin{aligned}
z & =v t+z_{k}, \\
r & =0 .
\end{aligned}
$$

The energy loss of this particle is obtained by integrating the force acting on it over the time taken to cross the cavity:

$$
W_{k}=e v \int_{-z_{k} / v}^{\left(g-z_{k}\right) / v} E_{z}\left(r=0, z=v t+z_{k}, t\right) \mathrm{d} t
$$

Since:

$$
E_{z}=-\frac{\partial \phi}{\partial z}-\frac{1}{c} \frac{\partial A_{z}}{\partial t}
$$

we obtain, using eqs. (38) to (40):

$$
W_{k}=e v \sum_{p=0}^{\infty} \int_{-\infty}^{+\infty} \frac{\mathrm{i} c \lambda^{2}}{\omega} A_{p}(\omega, 0) c_{p k}(\omega) \mathrm{d} \omega
$$

where:

$$
c_{p k}(\omega)=\int_{-z_{k} / v}^{\left(g-z_{k}\right) / v} \exp (-\mathrm{i} \omega t) \cos \left[\pi p\left(v t+z_{k}\right) / g\right] \mathrm{d} t .
$$

We now introduce an explicit expression for the beam current $I$ :

$$
I(z-v t)=e v \sum_{n=-\infty}^{+\infty} \sum_{k=1}^{N} \delta\left(z-v t-z_{k}-n v T\right) .
$$

Here, $T$ is the time interval between successive bunches, each containing $N$ particles. The sum over $n$ describes the passages of the bunch through the cavity. Using eq. (51), the quantity $c_{p}(\omega)$ defined in eq. (44) can be written as follows:

$$
c_{p}(\omega)=\frac{e v}{2 \pi} \sum_{n=-\infty}^{+\infty} \exp (-\mathrm{i} \omega n T) \sum_{k=1}^{N} c_{p k}(-\omega)
$$


Putting eq. (52) into eq. (46), and the latter into eq. (49), yields for the energy loss of the $k$ th particle:

$$
W_{k}=\frac{e^{2} v^{2}}{g} \sum_{p=0}^{\omega} \frac{1}{1+\delta_{p 0}} \int \mathrm{d} \omega P(\omega) \sum_{n=-,}^{+\infty} \exp (-\mathrm{i} \omega n T) \sum_{m=1}^{N} c_{p m}(-\omega) c_{p k}(\omega) .
$$

Here, we have omitted the term $H^{(1)}(\lambda r)$ in eq. (46) since it describes the self-field of the beam in vacuum, and introduced:

$$
P(\omega)=\frac{\lambda^{2}}{\omega} \frac{H_{0}^{(1)}(\lambda b)+(i \omega / c \lambda) \mathscr{R} H_{1}^{(1)}(\lambda b)}{J_{0}(\lambda b)+\left(i \omega /(\hat{\lambda}) \Re R J_{1}(\lambda b)\right.} .
$$

The average energy loss $W$ can be defined as:

$$
W=\frac{1}{N} \sum_{k=1}^{N} W_{k}
$$

It is immediately obvious from eqs. (53) to (55) that for $=0$, the average energy loss $W$ vanishes. For the case $\mathscr{R} \neq 0$, the average energy loss can be written as follows:

$$
w=\frac{e^{2} v^{2}}{2 N g} \sum_{p=0}^{\infty} \frac{1}{1+\delta_{p 0}} \int[P(\omega)+P(-\omega)] \sum_{n=-\infty}^{+\infty} \exp (-\mathrm{i} \omega n T) \sum_{k, m=1}^{N} c_{p m}(-\omega) c_{p h}(\omega) \mathrm{d} \omega .
$$

By using the relationship:

$$
J_{0}(z) H_{1}^{(1)}(z)-J_{1}(z) H_{0}^{(1)}(z)=-2 i / \pi z,
$$

and by evaluating $c_{p k}(\omega)$ from eq. (50), eq. (56) becomes:

$$
\begin{aligned}
W= & \frac{2 e^{2}}{\pi N b g\left(v^{2}\right.} \sum_{p=0}^{\infty} \frac{1}{1+\delta_{p 0}} \int \frac{\left(\not R+R^{*}\right) \omega^{2} \mathrm{~d} \omega}{\left[J_{0}(\lambda b)+\left(\mathrm{i} \omega \cdot \mathscr{R} /(\dot{\lambda}) J_{i}(\lambda b)\right]\left[J_{0}(\lambda b)-\left(\mathrm{i} \omega \cdot \mathscr{R} /(\lambda) J_{1}(\dot{\lambda} b)\right]\right.\right.} \frac{1-(-1)^{p} \cos (\omega g / v)}{\left[(\pi p / g)^{2}-(\omega / v)^{2}\right]^{2}} \\
& \times \sum_{n=0}^{\infty} \sum_{k, m=1}^{N} \exp \left[-\mathrm{i} \omega\left(n T+\frac{z_{k}-z_{m}}{v}\right)\right] .
\end{aligned}
$$

At this point, we may use two alternative ways to perform the integration over $\omega$. We may either exploit the periodicity of the bunch current driving the cavity, or we may integrate over $\omega$ in the complex plane, using the residue theorem which yields $W$ as a sum over the cavity resonant frequencies. The sums over $k$ and $m$ can subsequently be evaluated once the bunch shape is given. In the following, we shall assume a Gaussian bunch shape, replacing the sum over $k$ by an integral:

$$
\sum_{k=1}^{N} \rightarrow \frac{N}{(2 \pi)^{\frac{1}{2}} \Delta} \int_{-\infty}^{+\infty} \exp \left(-z_{k}^{2} / 2 \Delta^{2}\right) \mathrm{d} z_{k}
$$

Using the first way, we replace the sum over $n$ in eq. (57) by the Poisson sum formula:

$$
\sum_{n=-\infty}^{+\infty} \exp (-\mathrm{i} \omega n T)=\omega_{0} \sum_{n=-\infty}^{+\infty} \delta\left(\omega-n \omega_{0}\right)
$$

with $\omega_{0}=2 \pi / T$, and obtain:

$$
W=-\frac{8 e^{2} \omega_{0}^{3} N}{\pi b g v^{2} c} \sum_{n=0}^{s} \sum_{p=0}^{\infty} \frac{n^{2}}{1+\delta_{p 0}} \frac{1-(-1)^{p} \cos \left(n \omega_{0} g / v\right)}{\left[(\pi p / g)^{2}-\left(n \omega_{0} / v\right)^{2}\right]^{2}}\left(\frac{n \omega_{0}}{8 \pi \sigma}\right)^{\frac{1}{2}} D_{n p} \exp \left(-n^{2} \omega_{0}^{2} \Delta^{2} / v^{2}\right),
$$

where:

$$
D_{n p}^{-1}=\left[J_{0}\left(\lambda_{n p} b\right)-\frac{n \omega_{0}}{c \lambda_{n p}}\left(\frac{n \omega_{0}}{8 \pi \sigma}\right)^{1} J_{1}\left(\lambda_{n p} b\right)\right]^{2}+\left[\frac{n \omega_{0}}{c \dot{\lambda}_{n p}}\left(\frac{n \omega_{0}}{8 \pi \sigma}\right)^{1} J_{1}\left(\lambda_{n p} b\right)\right]^{2},
$$


and:

$$
\lambda_{n \rho}^{2}=\left(n \omega_{0} / c\right)^{2}-(\pi p / g)^{2} .
$$

Using the second way, namely the residue theorem, we find:

$$
W=\frac{8 e^{2} N}{g} \sum_{p=0}^{\infty} \sum_{s=1}^{\infty} \frac{R_{s p} \exp \left[-\left(\omega_{s p} \Delta / v\right)^{2}\right]}{\left(1+\delta_{p 0}\right) v_{s}^{2} J_{1}^{2}\left(v_{s}\right)} \frac{1-(-1)^{p} \cos \left(\omega_{s p} g / v\right)}{\left[1+\left(\pi p b / \gamma v_{s} g\right)^{2}\right]^{2}}
$$

The resonant factor $R_{s p}$ is:

$$
R_{s p}=\frac{\sinh \left(\pi \omega_{s p} / \omega_{0} Q_{s p}\right)}{\cosh \left(\pi \omega_{s p} / \omega_{0} Q_{s p}\right)-\cos \left(2 \pi \omega_{s p} / \omega_{0}\right)} .
$$

The resonant frequencies $\omega_{s p}$ are given by:

$$
\left(\omega_{s p} / c\right)^{2}=\left(v_{s} / b\right)^{2}+(\pi p / g)^{2},
$$

where $v_{s}$ is the $s$ th root of the equation $J_{0}\left(v_{s}\right)=0$. The quality factor is given by:

$$
Q_{s p}=(b / c)\left(2 \pi \sigma \omega_{s p}\right)^{\frac{1}{2}} .
$$

In evaluating eq. (63), the position of the poles has been determined to first order in $\mathscr{R}$, and all terms in $W$, except the resonant factor $R_{s p}$, have been evaluated for $\mathscr{R}=0$. 